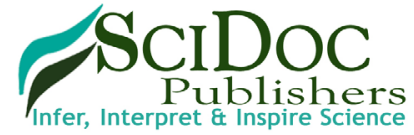

\section{Causes of Admission And Clinical Outcomes Among Adult ICU Admitted Patients at Wollega University Referral Hospital}

Nega Desalegn*

Assistant Professor of Anesthesiology, Department of Anesthesiology, Wollega University, Ethiopia.

\title{
Abstract
}

Background: Intensive care unit is a part of the hospital where critically ill patients who require advanced airway, respiratory, and hemodynamic unstable were usually admitted for continues monitoring and better outcome than if the patients were admitted to other wards of the hospital.Evaluation causes admission, causes of death and the outcomes of medical interventions will help to assess the efficacy of treatment, making it possible totake better decisions, to further improve quality of care, to standardize conduct, and to ensureeffective management of the high-level resources needed to deliver intensive care servicesthereby optimizing resource utilization.

Objective: The objective of this study is to assess the causes of admission and clinical outcomes of patients admitted to Wollega University Referral Hospital adult intensive care unit from September 2017 to September 2019.

Methodology: Retrospective review of ICU admitted patient's log books and clinical charts at Wollega University Referral Hospital adult Intensive Care Unitfrom September 2017 to September 2019.

Result: A total of 496 of the patient charts were reviewed; 271 (54.6\%) of the patients were males and 133(45.4\%) were females, a male: female ratio of 1.2:1. The median age at admission was 34 years with interquartile of 25-34 years and Standard deviation of 16.2. Meningitis (10.7\%), Bronchial Asthma (9.7\%), CHF (8.5\%), Stroke(8.1\%)andEclampsia (7.3\%) were the five commonest causes for ICU admission. The median length of ICU staywas two days and mortality rate of $29.0 \%$.

Conclusions: The mortality at the Adult intensive care unit was high (29.0\%). Meningitis and Bronchial asthma were main causes for ICU admission while Multi organ failure, brain death and respiratory failure were the main causes of death.

Keywords: Causes of Admission; Death; Adult ICU; Outcome.

\section{Introduction}

In developing countries, resources are scarce and provisionof ICU care is very challenging. In Ethiopia, ICU care has been introduced only a few decades ago. The number of ICUs is increasing recently in government and private institutions across the country. However, the care delivered at the ICUs in the country has remained largely unstudied. This study aimed to assess the causes of admission, length of stay and treatment outcomes in Wollega University referral hospital Adult ICU. The results are compared with the current practice within Ethiopia and other settings.

An evaluation of the outcomes and hospital morbidity pattern is an important measure to guide improvements in patient care. In intensive care units (ICUs), the success of care can best be meas- ured by the out- comes such as survival or death among those admitted to the unit by using indicators such the proportion of deaths [11]. One should be aware of the prevalent conditions and set up the facilities and prepare treatment. The mean ICU stay was $4.9 \pm 5.8$ days (range 1-30 days) vast majority of patients admitted to ICU were admitted medical of which meningitis (44/400 pts., $11 \%$ ), post-operative (43/400 pts., $10.8 \%$ ) [2].

The number of cases with percentage of Hepatic diseases $12.9 \%$, CCF and Pulmonary edema 6.2\%, Cellulitis 0.8\%, CVA $11.6 \%$, Hypertensive Crisis 4.1\%, Arrhythmias $0.8 \%$, Diabetes with complication $2.5 \%$, Carcinoma $0.4 \%, 2.1 \%$ Tb with complication, Respiratory issues $9.5 \%$, ACS 5.4\%, Kidney diseases 5.4\%, Sepsis $10.4 \%$, GI Bleed 3.3\%, Heat stroke $0.4 \%$, CNS Infection $7.9 \%$, Poisoning $3.3 \%$, Shock $1.2 \%$, AGE with complication $5.8 \%$, Sur-

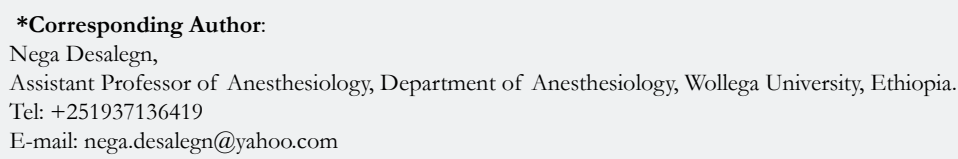

Citation: Nega Desalegn. Causes of Admission And Clinical Outcomes Among Adult ICU Admitted Patients at Wollega University Referral Hospital. Int J Anesth Res. 2021;09(03):636641. doi: http://dx.doi.org/10.19070/2332-2780-21000126

Copyright: Nega Desalegn ${ }^{\circ}$ 2021. This is an open-access article distributed under the terms of the Creative Commons Attribution License, which permits unrestricted use, distribution and reproduction in any medium, provided the original author and source are credited. 
gical and Gynecological issues were 5.4\% and $0.4 \%$ (related to medical issues).[8]

Among the adult patients, there were $23(31 \%)$ patients with trauma. There were $45(61 \%)$ surgical patients and $6(8 \%)$ medical patients. Sixteen $(19 \%)$ patients were mechanically ventilated. The overall mortality was $28(32.9 \%)$. Sixty-four percent of the mortality occurred during the first 24 hours of admission. A mortality rate of $83.3 \%$ was recorded among medical patients and $62.5 \%$ in those referred from the wards [4].

In Ethiopia previous study shows which is done black lion teaching hospital in patterns of medical admission in toMedical ICU from 1985-2000 acute infection and cardiovascular disease accounted for half of the entire critical care admission with infectious disease accounted for 30\% among specific diagnose diabetic ketoacidosis was the leading cause of admission followed by acute myocardial infection and severe and complicated malaria each accounting for (9.3) of all admission respectively.[3]

This study will provide a baseline data for future reviews, health workers and planners to give due attention to improving the outcome of care in critically ill patients as there is no previous study on this topic in this setting.

This study aims to assess the causes of admission and outcome of adult patients managed at the Adult intensive care unit of Wollega University Referral Hospital.

\section{Methodology}

Wollega University Referral Hospital (WURH) is the largest referral hospital in western Ethiopia. It started as a referral and teaching University hospital in 2017, providing service for about 5 million people. A two years retrospective cross sectional study was conducted in 8 bedded Adult ICU from September 2017 to September 2019 and the data was collected from ICU logbook and patient charts/cards.

\section{Sample size and sampling techniques}

All consecutively admitted patients to AICU from September 2017 to September t 2019 were included, based on the Inclusion and exclusion criteria. All patients admitted to AICU whose age is greater than 15 yearswere included in the study andpatients with incomplete or missed data were excluded from the study. Other patients excluded from the study were, those who died on arrival (within $2 \mathrm{~h}$ of admission); this is not sufficient time to give optimal care in the ICU, and because the outcome of these patients is related to the emergency or other ward care.

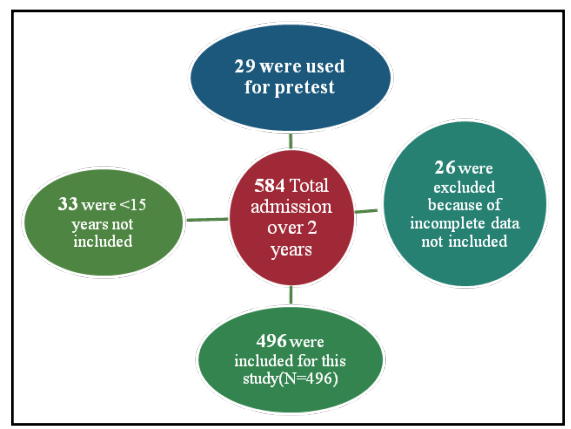

\section{Study variable}

\section{Dependent variable}

Clinical outcome

\section{Independent variable}

Age, sex, length of ICU stay, diagnosis at admission, inotropes use, comorbid morbidities.

Admission sources, Glasgow Coma Scale (GCS) at admission, Blood pressure, Pulse rate, urine output, temperature on admission, blood transfusion, complication of blood transfusion, time of patient death, use of admission criteria , use weaning criteria, use of feeding guide, who admitted the patient, patient out come and causes of patient death.

\section{Data collection}

The questionnaire containing the above variables was adapted by reviewing different similar literature. Two BSc anesthetists were hiredfor the data collection after one day training was given to them on the objectives of the study, the contents of the questionnaire how to fill the questionnaire and on issues related to the confidentiality.The principal investigator was continuously supervised the data collectors for completeness and consistency. Prior to the data collection, a pre-test was conducted at WURH on $5 \%$ (29 patient cards) of the sample size for completeness of the questionnaire and based on the findings of the pre-test, questionnaire was modified.

\section{Data analysis}

Data entry and analysis was done using SPSS software (version 25.0). The generated data compiled by frequency table, charts and graphs. Statistical significancewas considered at a $\mathrm{p}$ value of $<$ 0.05.where appropriate to use.

\section{Ethical considerations}

Prior to data collection, ethical approval was obtained from ethical review committee of Wollega University and WURH card room workers were informed about the purpose of the study and they were given a letter written by the Hospital director to give access to the cards identified from ICU logbook based on the study period.

\section{Results}

A total of 496 of the patient charts were reviewed; $271(54.6 \%)$ of the patients were males and 133(45.4\%) were females, a male: female ratio of 1.2:1. The median age at admission was 34 years with interquartile of 25-34 years and those $>65$ years accounts only $5 \%$ of the admission, regarding the ICU outcome $29.1 \%$ ( 144) death, $61.9 \%$ (307) improved while about $1.6 \%(8)$ self-discharged.(Table 1)

The main causes of admission to WURH Adult ICU were Meningitis 10.7\%, Bronchial Asthma 9.7\%, CHF 8.5\%, stroke $8.1 \%$, 
Trauma 7.3\% Other $9.9 \%$ (other includes :Uterine rupture, UTI, Suicidal attempt, Poisoning, esophageal cancer, Aspiration pneumonia.

Regarding patient source for ICU admission Emergency OPD accounts $65.3 \%$ and Operation theatre and Medical ward accounts for $15.3 \%, 14.1 \%$ respectively (Table 2 ). And $48.0 \%$ stayed less than 2 days while only $3.6 \%$ stayed $>10$ days.
$48.4 \%(74)$ of those stayed less than 2 days has died while 33.3 $\%(4)$ of those stayed $>10$ days died and there is association between the length of hospital stay and patient outcome $(\mathrm{p}<0.001)$ (Table 1).34.9\% of the patient admitted to ICU from the emergency OPD and $15.7 \%$ from operation theatre were died while no death rate among the patients admitted from surgical ward (Table1) and there is association among the patient source (departments) and their ICU outcome. $(\mathrm{p}<0.001)$.

Figure 1. Causes of ICU admission.

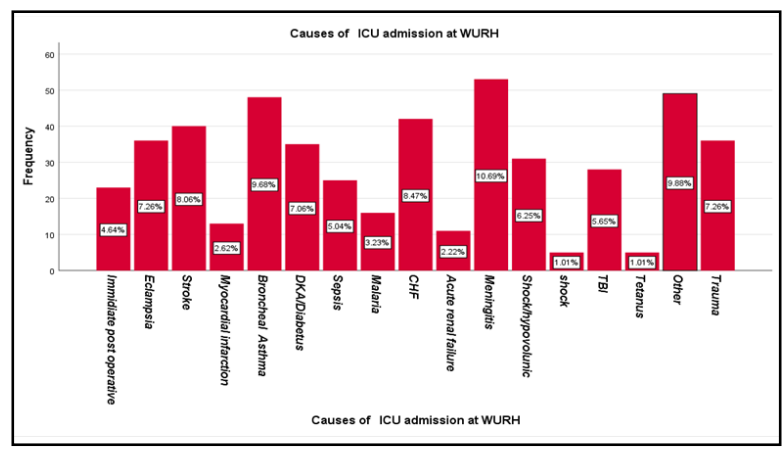

Figure 2. Causes of patient death in ICU.

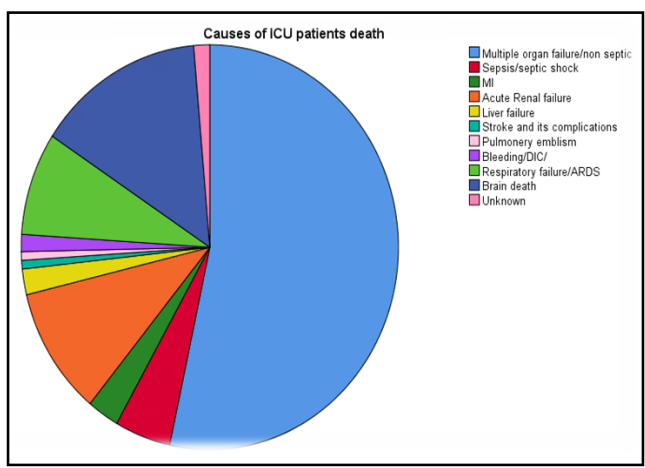

Table 1. Gender Age, length of ICU stay, Patient source, Conscious status and patients outcome.

\begin{tabular}{|c|c|c|c|c|c|c|c|}
\hline & & \multicolumn{5}{|c|}{ Patients out come } & \multirow{2}{*}{ Pvalue } \\
\hline & & Improved & Death & Referred & Self-discharge & Total & \\
\hline \multirow{5}{*}{ Patients length of ICU stay } & $<2$ days & $153(64.3 \%)$ & $74(31.1 \%)$ & $9(3.7 \%)$ & $2(0.84 \%)$ & $238(48.0 \%)$ & \multirow{5}{*}{$\mathrm{P}<0.001$} \\
\hline & 2-5 days & $77(58.3 \%)$ & $43(32.6 \%)$ & $10(7.6 \%)$ & $2(1.5 \%)$ & $132(26.6 \%)$ & \\
\hline & 5-10 days & $65(60.2 \%)$ & $23(21.3 \%)$ & $18(16.7 \%)$ & $2(1.85 \%)$ & $108(21.8 \%)$ & \\
\hline & $>10$ days & $12(66.7 \%)$ & $4(22.2 \%)$ & $0(0.0 \%)$ & $2(11.1 \%)$ & $18(3.6 \%)$ & \\
\hline & Total & $307(61.9 \%)$ & $144(29.0 \%)$ & $37(7.5 \%)$ & $8(1.6 \%)$ & 496 & \\
\hline \multirow{3}{*}{ Gender } & Male & $171(63.1 \%)$ & $78(28.8 \%)$ & $17(6.3 \%)$ & $5(1.8 \%)$ & $271(54.6 \%)$ & \\
\hline & Female & $136(60.4 \%)$ & $66(29.3 \%)$ & $20(8.9 \%)$ & $3(1.3 \%)$ & $225(45.4 \%)$ & $\mathrm{P}=0.68$ \\
\hline & Total & $307(61.9 \%)$ & $144(29.0 \%)$ & $37(7.5 \%)$ & $8(1.6 \%)$ & 496 & \\
\hline \multirow{7}{*}{ Age distribution } & $16-25$ & $110(68.8 \%)$ & $40(25.0 \%)$ & $10(6.25 \%)$ & $0(0.0 \%)$ & $160(32.3 \%)$ & \\
\hline & $26-35$ & $63(53.4 \%)$ & $46(40.0 \%)$ & $9(7.6 \%)$ & $0(0.0 \%)$ & $118((23.8 \%)$ & \\
\hline & $36-45$ & $52(72.2 \%)$ & $12(16.7 \%)$ & $4(5.6 \%)$ & $4(5.6 \%)$ & $72(14.5 \%)$ & \\
\hline & $46-55$ & $37(59.7 \%)$ & $18(29.0 \%)$ & $5(8.1 \%)$ & $2(3.2 \%)$ & $62(12.5 \%)$ & $\mathrm{P}=0.001$ \\
\hline & $56-65$ & $35(59.3 \%)$ & $19(32.2 \%)$ & $5(8.5 \%)$ & $0(0.0 \%)$ & $59(11.9 \%)$ & \\
\hline & $>65$ & $10(40.0 \%)$ & $9(36.0)$ & $4(16.0 \%)$ & $2(8.0 \%)$ & $25(10.5 \%)$ & \\
\hline & Total & $307(61.9 \%)$ & $144(29.0 \%)$ & $37(7.5 \%)$ & $8(1.6 \%)$ & 496 & \\
\hline \multirow{7}{*}{$\begin{array}{c}\text { Source of patient for ICU } \\
\text { Admission }\end{array}$} & Operation theatre & $63(82.9 \%)$ & $12(15.8 \%)$ & $1(1.3 \%)$ & $0(0.0 \%)$ & $76(15.3 \%)$ & \\
\hline & Medical ward & $47(67.1 \%)$ & $17(24.3 \%)$ & $5(7.1 \%)$ & $1(1.4 \%)$ & $70(14.1 \%)$ & \\
\hline & Emergency OPD & $174(53.7 \%)$ & $113(34.9 \%)$ & $30(9.3 \%)$ & $7(2.2 \%)$ & $324(65.3 \%)$ & \\
\hline & Surgical ward & $4(100.0 \%)$ & $0(0.0 \%)$ & $0(0.0 \%)$ & $0(0.0 \%)$ & $4(0.8 \%)$ & \\
\hline & Obstetric/GYN Ward & $19(86.4 \%)$ & $2(9.0 \%)$ & $1(4.5 \%)$ & $0(0.0 \%)$ & $22(4.4 \%)$ & \\
\hline & Total & $307(61.9 \%)$ & $144(29.0 \%)$ & $37(7.5 \%)$ & $8(1.6 \%)$ & 496 & \\
\hline & Conscious & $204(73.9 \%)$ & $62(22.5 \%)$ & $8(2.9 \%)$ & $2(0.7 \%)$ & $276(55.6 \%)$ & \\
\hline \multirow[t]{3}{*}{$\begin{array}{c}\text { Conscious status on ICU } \\
\text { admission } \\
\end{array}$} & Unconscious & $96(45.0 \%)$ & $82(34.5 \%)$ & $29(13.6 \%)$ & $6(2.8 \%)$ & $213(42.9 \%)$ & \\
\hline & Unknown & $7(100 \%)$ & $0(0.0 \%)$ & $0(0.0 \%)$ & $0(0.0 \%)$ & $7(1.4 \%)$ & \\
\hline & Total & $307(61.9 \%)$ & $144(29.0 \%)$ & $37(7.5 \%)$ & $8(1.6 \%)$ & 496 & \\
\hline
\end{tabular}


Table 2. Distribution of coexisting disease among ICU admitted patients.

\begin{tabular}{|c|c|c|}
\hline Coexisting disease & Frequency & Percent \\
\hline CVS disease & 121 & 24.4 \\
\hline Respiratory disease & 16 & 3.2 \\
\hline Infection & 29 & 5.8 \\
\hline Neurologic disorder & 7 & 1.4 \\
\hline Endocrine disease & 9 & 1.8 \\
\hline No coexisting disease & 209 & 42.1 \\
\hline Hypertension & 90 & 18.1 \\
\hline Other & 15 & 3 \\
\hline Total & 496 & 100 \\
\hline
\end{tabular}

CVS disease $(24.4 \%)$ is the main coexisting disease ICU admitted patients have and $42.1 \%$ has no coexisting disease.

$22.5 \%$ of those conscious on admission were died while $38.5 \%$ of those unconscious on admission where died and there is statistically significant association among patient's conscious status on admission and their ICU outcome $\mathrm{p}$ value $<0.001$ (Table 3 above).

Regarding blood transfusion in ICU only 3.6\% (18) patients received PRBC and $16.7 \%(3)$ of those transfused were died.

The common vasopressors used were adrenaline and dopamine; they were used for 139 patients of which 36.0\% Adrenaline and $64.0 \%$ dopamine; $46.0 \%$ (23) treated with adrenaline and $37.1 \%(33)$ treated with dopamine were died.

Multiple organ failure $53.4 \%$, Brain death $14.4 \%$, Acute renal failure $10.3 \%$, Respiratory failure $8.2 \%$ and sepsis/septic shock $4.8 \%$ were the five major causes of ICU patient death in this setting.

\section{Discussion}

An Intensive Care Unit (ICU), is a specialized section of a hospital that provides comprehensive and continuous care for persons who are critically ill and who can benefit from treatment. Patients are generally admitted to an ICU if they are likely to benefit from the level of provided care. Intensive care has been shown to be beneficial for patients who are severely ill and medically unstable that is, theyhave a potentially life threatening disease or disorderAbout one third of hospital mortality occurs /in critically ill patients inside Intensive Care Unit (ICU) [7].

In this study setting we have used age lowest limit 16years because there are also some patients admitted to ICU with age $<15$ years and those where currently managed in separate pediatric ICU and also similar studies used 16years as start age of adult cases.

In this setting there were about two mechanical ventilators during the study period and no patients were mechanically ventilated because of malfunction (biomedical engineers warned not to use) and currently there are about eight functional mechanical ventilators for eight bedded Adult ICU and three other functional mechanical ventilators for six bedded pediatric ICU.

Majority of the patients were admitted by senior physician evaluation without ICU consultant critical care specialist or anesthesiologist and there is no admission and discharge criteria consid- eration to admit or discharge the patients, similarly there is no feeding guideline use in this setting.

In this study majority of the patients were males $(54.6 \%)$ while females accounts about (45.4\%) which is similar with the one year retrospective study done at Jimma university medical center 134 $(53.17 \%)$ were males and $118(46.38 \%)$ females[9] and there is no association between gender and patient outcome $(p=0.68)$.

The median age was 34 with interquartile of 25-34 years, majority of ICU admitted cases where less than 25 years accounts $160(32.3 \%)$ while $25-35$ years and there is association between age and patient outcome $(\mathrm{p}<0.001)$.

Sources patient for admissions Emergency department majority of causes accounts $324(65.3 \%)$ the flowed by operation theatre $76(15.3 \%)$ and medical wards $70(14.1 \%)$ while the retrospective study done at the University of Calabar Teaching Hospital, Nigeria where $64(75.3 \%)$ patients from the operating room, $8(9.4 \%)$ from the inpatient wards, and 13 (15.3\%) from the accident and emergency department.

Regarding mortality rate it is about $29 \%$ which is similar to the mortality rateCalabar Teaching Hospital, Nigeria which is mortality of $28(32.9 \%)$ [4]. and other study in najeria teaching hospital [7] in this study setting which is $29.0 \%$.("Abstract," n.d.)) One year retrospective study done at Jimma university medical center ICU showed mortality rate of $50.4 \%$ where traumais the leading admission cause accounting about $(19.2 \%)$ total admission but in our setting trauma accounts only (7.2\%) [9] and this is much bigger than our setting and ICU mortality varies across the world depend on ICU infrastructure, staff availability, and training, pattern, and cause of ICU admission [1].

In developed contents like North America, Oceania, Asia and Europe in ICU mortality relatively low with the rate of $9.3 \%, 10.3$, $13.7 \%$ and $18.7 \%$ respectively, while in the Middle East the mortality found to be $21.7 \%$ and $26.2 \%$ [14] and the study conducted at hospital of Sergipe, northeastern Brazilidentified a $21 \%$ ICU mortality rate which is comparable with this study finding [10].

Regarding length of ICU stay majority $238(48.0 \%)$ stayed less than two days while $132(26.6 \%)$ stayed $2-5$ days and $18(3.6 \%)$ stayed greater than ten days with median ICU stay oftwo days and 
interquartile of 1-2 days and there is association between length of ICU stay and patient outcome $(\mathrm{p}<0.001)$; while the study done at University of Gondar where median length of ICU stay (IQR) was $4(2-5)$ days. The most frequent stay $(19.8 \%)$ was 1 day and cardiogenic shock and ARDS accounts (57.1\%) (54.4\%) respectively, [12] the median stay on mechanical ventilator is 4.5 days the difference may be due to mechanical ventilator use for the above main causes of admission. This study finding is similar to study done at Jimma medical center showed median length-of-stay (interquartile range) was $3.0(1.0-7.0)$ days.[9]

Regarding the conscious status on ICU admission about 213 (42.9\%)Unconscious among this $82(34.5 \%)$ were died and there is association between conscious status on admission and patient outcome $(p<0.05)$; disturbance level of consciousness is related with severe decompensated disease, cerebral hypo-perfusion due to sepsis, blood loss, poisoning, and neurological disorder .

The main causes of admission to ICU at this study setting were Meningitis 10.7\%, Bronchial Asthma 9.7\%,CHF 8.5\%, stroke 8.1\% , Trauma 7.3\%; while the study done at Armed Force General Teaching Hospital Ethiopia showed TBI had the major admission accounts for 25(8.9\%), DKA, 18(6.3\%) and MI 17(6.1\%), [3] the study done at Ayder hospital(medical ICU) Ethiopia shows (DKA), constituting $194(16.0 \%)$ of all admissions. Heart failure accounted for $193(16 \%)$, all types of stroke for $185(15.2 \%)$, myocardial infarction for $119(9.8 \%)$ and septic shock for $61(5 \%)$ of the admissions. [5] Cardiovascular, Respiratory Neurologic and Digestive193 (28) 182 (26) 169 (24) 135 respectively where the main causes of ICU admission [13].

Similar studies at university of Gondar shows Cardiovascular disease accounted $36 \%$ of all ICU admission followed by respiratory $(17.9 \%)$ which is similar to the studies done in Mekelle and Jimma [9] However, it was different from studies done in Uganda and Nigeria, infectious illness and post-operative care were the main reasons for admission [12] similar study done at Bahrain shows on Causes of Mortality among Critically Ill Patients Admitted in Intensive Care Unit.

Mohammed Cerebro vascular Accident (CVA) 100 (19.96\%) Renal Failure.

$99(19.76 \%)$ Myocardial Infarction (MI) 60 (11.97\% Malignancy34 (6.78\%) Hepatic Encephalopathy 32 (6.38\%) [6].

The common vasopressors used were adrenaline and dopamine; they were used for 139 patients of which $36.0 \%$ Adrenaline and $64.0 \%$ dopamine; $46.0 \%$ (23) treated with adrenaline and $37.1 \%$ (33) treated with dopamine were died.

Regarding blood transfusion in ICU only 3.6\% (18) patients received PRBC and 3(16.7\%)ofthose transfused were died.

Time of death, there is no association between the time of death and patient outcome $76(52.8 \%)$ died day time while $68(47.2 \%)$ died at night.

Regarding coexisting disease of ICU admitted patients Cardiovascular disease (24.4\%), Hypertension $(18.1 \%)$ were the two common coexisting disease and about $(42.1 \%)$ has no coexisting disease and other study shows CVS disease $26 \%$, respiratory $27 \%$ and infection $13 \%$ [12] which is almost similar to this study setting .

Multiple organ failure 53.4\%, Brain death $14.4 \%$, Acute renal failure $10.3 \%$, Respiratory failure $8.2 \%$ and sepsis/septic shock $4.8 \%$ were the five major causes of ICU patient death in this setting. Prospective Multicenter Study done on Causes and Characteristics of Death in Intensive Care Units: total of 698 patients were included during the study period. At the time of death, $84 \%$ had one or more organ failures (mainly hemodynamic) and $89 \%$ required at least one organ support (mainly mechanical ventilation). [13] similar study at Bahrain shows Circulatory System Disorders 1215 (42.91\%) Renal Failure 102 (20.36\%) Malignancy235 (6.99 \%) Infectious Diseases 34 (6.79\%) Hepatic Encephalopathy 32 $(6.39 \%)$ Head Injury 25 (4.99\%) [6].

\section{Conclusion and Recommendation}

Meningitis and bronchial asthma were among the main causes of admission while multiple organ failure is the main causes of death Mortality rate was $29.0 \%$ and the time of death (day/night) has no association with the patient outcome.

There is no admission, discharge and feeding guideline on use in this setting which resulted unnecessary ICU admission and bed saturation.

This settingneed to used standard guidelines for patient safety and effective resource use.

\section{Limitation of the study}

The limitations of this study include the retrospective design; the mortality rate may be falsely low in resources limited setting due to sample size.

\section{Competing interests}

The authors declare that there is no competing interest.

\section{Acknowledgement}

The authors are thankful for all participants and no funding received during the conduct of this study.

\section{References}

[1]. Agalu A, Woldie M, Ayele Y, Bedada W. Reasons for admission and mortalities following admissions in the intensive care unit of a specialized hospital, in Ethiopia. Int J Med Med Sci. 2014 Sep 30;6(9):195-200.

[2]. Haftu H, Hailu T, Medhaniye A. Assessment of pattern and treatment outcome of patients admitted to pediatric intensive care unit, Ayder Referral Hospital, Tigray, Ethiopia, 2015. BMC research notes. 2018 Dec;11(1):1-6.

[3]. Haile S. One Year Retrospective Review of Disease Patterns and Clinical Outcomes of Patients Admitted in Intensive Care Units of Armed Force General Teaching Hospital in Addis Ababa, Ethiopia (Doctoral dissertation, Addis Ababa University).

[4]. Ilori IU, Kalu QN. Intensive care admissions and outcome at the University of Calabar Teaching Hospital, Nigeria. J Crit Care. 2012 Feb;27(1):105. e1-4. Pubmed PMID: 22304993.

[5]. Gidey K, Hailu A, Bayray A. Pattern and outcome of medical intensive care unit admissions to ayder comprehensive specialized hospital in tigray, ethiopia. Ethiopian Medical Journal. 2018;56(1).

[6]. Omar MA, Aram FO, Banafa NS. Causes of mortality among critically ill patients admitted in intensive care unit. Bahrain Medical Bulletin. 2015 
Sep;37(3):178-80.

[7]. Onyekwulu FA, Anya SU. Pattern of admission and outcome of patients admitted into the Intensive Care Unit of University of Nigeria Teaching Hospital Enugu: A 5-year review. Niger J Clin Pract. 2015 Nov-Dec;18(6):775-9. Pubmed PMID: 26289516.

[8]. Rajput T, Sohail F, Qabulio SN, Zakir MF, Soomro MY. Admission Patterns and Outcomes in an Adult Intensive Care Unit in Medical Patients in Karachi.

[9]. Smith ZA, Ayele Y, McDonald P. Outcomes in critical care delivery at Jimma University Specialised Hospital, Ethiopia. Anaesth Intensive Care. 2013 May;41(3):363-8. Pubmed PMID: 23659399.

[10]. Soares Pinheiro FGM, Santana Santos E, Barreto ÍDC, Weiss C, Vaez AC, Oliveira JC, et al. Mortality Predictors and Associated Factors in Patients in the Intensive Care Unit: A Cross-Sectional Study. Crit Care Res Pract. 2020 Aug 1;2020:1483827. Pubmed PMID: 32802502.

[11]. azebew, Ashenafi, Tigist Bacha Heye, Ashenafi Tazebew, Biniyam Chakilu
Tilahun, and Tigist Bacha Heye. "ORIGNIAL ARTICLE ADMISSION PATTERN AND OUTCOME IN A PEDIATRIC INTENSIVE CARE UNIT OF GONDAR UNIVERSITY HOSPITAL” 2019;57 (2): 1-5.

[12]. Lema GF, Tessema HG, Mesfin N, Fentie DY, Arefaynie NR. Admission pattern, Clinical outcomes and associated factors among patients admitted in medical intensive care unit at University of Gondar Comprehensive and specialized hospital, Northwest Ethiopia, 2019. A retrospective crosssectional study.

[13]. Orban JC, Walrave Y, Mongardon N, Allaouchiche B, Argaud L, Aubrun F, et al. Causes and Characteristics of Death in Intensive Care Units: A Prospective Multicenter Study. Anesthesiology. 2017 May;126(5):882-889. Pubmed PMID: 28296682.

[14]. Vincent JL, Marshall JC, Namendys-Silva SA, François B, Martin-Loeches I, Lipman J, et al. Assessment of the worldwide burden of critical illness: the intensive care over nations (ICON) audit. Lancet Respir Med. 2014 May;2(5):380-6. Pubmed PMID: 24740011. 